\title{
Intercambio cooperativo versus mercantilización competitiva: las políticas de movilidad académica en el MERCOSUR y la Unión Europea'
}

Facundo Solanas

\section{RESUMEN}

La génesis de los procesos de internacionalización de la educación superior se vinculó estrechamente a la cooperación académica y científica entre las universidades, aunque el auge de la globalización y los procesos de mercantilización del sector transformaron en buena medida a ese intercambio en competitividad y competencia. Tanto el proceso de Bolonia como buena parte de las acciones de la Unión Europea (UE) pensadas para otros continentes, se enmarcan dentro de la estrategia para el mercado interior de servicios del Consejo Europeo de Lisboa de 2000, buscando una Europa más competitiva, donde el principal interés apuntaba a ampliar su "oferta" universitaria hacia los estudiantes de estos países. En este trabajo nos proponemos analizar cómo ha repercutido el cambio de paradigma sobre las políticas de movilidad académica en el MERCOSUR y la UE, apoyándonos en fuentes documentales y en la literatura existente en la materia.

Palabras clave: educación superior, movilidad académica, MERCOSUR, MERCOSUL, Unión Europea.

Facundo Solanas

fsolanas@yahoo.com

Argentino. Doctor en Ciencias Sociales por la Universidad de Buenos Aires (UBA). Investigador asistente del Consejo Nacional de Investigaciones Científicas y Técnicas (CONICET) y del Instituto de Investigaciones Gino Germani de la Facultad de Ciencias Sociales de la UBA. Profesor adjunto de la carrera de Sociología de la Facultad de Humanidades de la Universidad Nacional de Mar del Plata. Temas de investigación: políticas e industrias culturales, políticas de educación superior, políticas públicas comparadas entre procesos de integración regional, en particular en el MERCOSUR y la Unión Europea; sociología de los grupos profesionales, en particular los diplomáticos como cuerpo profesional.

${ }^{1} \mathrm{El}$ autor agradece las críticas y comentarios de los pares evaluadores anónimos que le han permitido mejorar el presente artículo. 


\title{
Intercâmbio cooperativo versus mercantilização competitiva: as políticas de mobilidade acadêmica no MERCosUL e na União Europeia
}

\section{RESUMO}

A gênese dos processos de internacionalização da educação superior ligava-se de maneira estreita com a cooperação acadêmica e científica dentre as universidades, embora o auge da globalização e os processos de mercantilização do setor tornaram, em grande medida, esse intercâmbio em competitividade e concorrência. Tanto o processo de Bolonha quanto grande parte das ações da União Europeia (UE) pensadas para outros continentes enquadram na estratégia para o mercado interior de serviços do Conselho Europeu de Lisboa de 2000, visando a uma Europa mais competitiva, onde o principal interesse apontava a aumentar a "oferta" universitária para estudantes desses países. Este trabalho visa analisar como é que a mudança de paradigma tem repercutido nas políticas de mobilidade acadêmica no MERCOSUL e na UE, com base em fontes documentais e na literatura existente na matéria.

Palavras chave: educação superior, mobilidade acadêmica, MERCOSUR, MERCOSUL, União Europeia.

\section{Cooperative exchange vs. competitive commodification: policies of academic mobility in MERCOSUR and the European Union}

\begin{abstract}
Originally, processes of internationalization for higher education were closely tied to academic and scientific cooperation between universities, although to a large degree the globalization boom and the sector's commodification processes transformed this exchange into competitiveness and competition. Both the Bologna process and a good part of the European Union's actions conceived for other continents are framed within the strategy for the internal services market of the European Council of Lisbon in 2000, seeking a more competitive Europe, where the main interest was geared towards broadening their university "supply" for students in those countries. In this work we analyze the repercussions of the shift in paradigms of academic mobility policies in MERCOSUR and the EU, based on documentary sources and existing literature on the subject.
\end{abstract}

Key words: higher education, academic mobility, MERCOSUR, MERCOSUL, European Union.

Recepción: 24/10/12. Aprobación: 24/06/13. 


\section{Introducción}

La movilidad internacional de estudiantes, docentes e investigadores no es un fenómeno novedoso. En especial, desde la segunda mitad del siglo XX, se fueron acentuando estos movimientos y en particular durante los últimos 30 años. Ahora bien, tanto las razones que impulsaron esta movilidad, como los factores que actualmente aumentan el flujo académico transfronterizo, no son precisamente las mismas.

A partir del auge del neoliberalismo en los años noventa, se profundizaron las dinámicas de internacionalización (Didou, 2006 y 2007; Musselin, 2008; Hermo y Pittelli, 2008; Haug, 2010) y mercantilización (Saforcada, 2009; Leher, 2009; Meabe y Saguier, 2011) de los sistemas de educación superior (SES). Esta tendencia se identifica fundamentalmente como consecuencia de tres factores interrelacionados, sobre los cuales nos detendremos: en primer lugar, el crecimiento de la "oferta" privada de educación superior y la proximidad entre éste ámbito y el mundo de los negocios (Robertson y Dale, 2003; Plotkin, 2006; Robertson, 2009; Rama, 2012); en segundo lugar, las regulaciones internacionales en el seno de la Organización Mundial de Comercio (OMC) y el Acuerdo General sobre Comercio y Servicios (AGCS) y sus esfuerzos por considerar la educación superior como un servicio (Kelsey, 2003; Robertson, 2003; Solanas, 2009b; Verger, 2010), y por último, la multiplicación de acuerdos entre instituciones de educación superior (IES), entre los cuales se incluyen los intercambios y programas de movilidad de docentes y estudiantes (Aboites, 2010; Bhandari, Blumenthal, 2011).

A su vez, estos factores han ido acompañados del desarrollo de un conjunto de políticas e instrumentos de políticas públicas, en su mayor parte impulsados por organismos internacionales, tales como: los cambios normativos en distintos países de los marcos reguladores de la educación superior (Chiroleu, 2000; Solanas, 2009a), la aparición de agencias de acreditación y evaluación universitaria (Marquina, 2006; Krotsch, Camou, Prati, 2007; Solanas, 2012), la aparición de distintos rankings internacionales de medición de la calidad de las universidades (Marginson y Wende, 2007; Linch, 2011), las estrategias desarrolladas por los distintos países y continentes para "captar" estudiantes internacionales (Robertson, 2009; Pepin, 2011), la traducción en "créditos" (una palabra muy económica) de los contenidos de la enseñanza superior, entre otros.

El desarrollo de las políticas de movilidad académica por parte de la Unión Europea (UE) a partir de los años ochenta, trajo aparejada la construcción de algunos instrumentos como el Sistema Europeo de Transferencia de Créditos (ECTS), sobre los cuales se asentaría "técnicamente" el proceso de Bolonia a fines de los años noventa. Este proceso, acompañado por la estrategia económica de Lisboa en busca de una Europa más competitiva, cuenta con una buena acogida por la mayor parte de las universidades y su territorio se expande hacia los países vecinos del continente (no ya sólo de la UE). Si bien América Latina no se encuentra comprendida directamente por este proceso, ha sido beneficiada de diversos programas de movilidad académica financiados por la UE como el ALFA, ALBAN y Tuning. Partimos de la hipótesis de que si bien ambas regiones se encuentran atravesadas por estas tendencias mercantilizantes de la educación superior, en el caso europeo y la movilidad académica, la impronta resulta ser mucho más notoria que en caso del Mercado Común del Sur (MERCOSUR). Ésta se traduce a través de las principales políticas de movilidad estudiantil europeas para el resto del mundo y su focalización en la necesidad de cooptar recursos humanos calificados para retenerlos en el continente, mientras que, por su parte, en el caso del MERCOSUR y América Latina, como muestra de resistencia a las tendencias mercantilizantes, ha habido un claro rechazo a la concepción de la educación superior como un "servicio" pregonada por la OMC. A su vez, los programas propios de movilidad - sin duda mucho menos desarrollados que en el caso europeo- se sustentan sobre las bases "tradicionales" 
de la cooperación internacional. Si bien en este trabajo el acento estará puesto en la movilidad de estudiantes, dado que constituye la más masiva, la misma tendencia también puede apreciarse sobre la movilidad de docentes e investigadores.

Apoyándonos en herramientas teóricas provenientes de la sociología de la acción pública y recurriendo al análisis de fuentes documentales, de la literatura existente en la materia, así como en distintas entrevistas realizadas con informantes clave, en este trabajo abordaremos la forma en que incrementalmente se manifiesta un cambio de paradigma del intercambio universitario hacia la competitividad entre las IES, en especial en la UE, pero cuyas consecuencias se expanden a América Latina y al MERCOSUR. Para ello, teniendo en cuenta diversos fenómenos interrelacionados, donde se manifiesta ese cambio de paradigma, analizaremos en primer lugar algunas de las características importantes sobre los procesos de internacionalización de la educación superior. En segundo lugar, tendremos en cuenta algunos de los principales elementos que ponen de manifiesto la mercantilización y la competitividad en la educación superior. Cabe aclarar que si bien cada uno de estos elementos, por sí solo bastaría para ser motivo de un artículo, no podemos dejar de mencionarlos, habida cuenta del impacto directo e indirecto que tienen sobre la movilidad académica. En tercer lugar, analizaremos el papel central de algunos organismos internacionales como la OMC y la concepción de la educación como un servicio. En cuarto lugar, abordaremos el auge de los programas de movilidad de estudiantes, en especial en la UE, como una de las aristas de la internacionalización y el cambio operado a partir del Proceso de Bolonia, así como sus consecuencias para América Latina y el MERCOSUR. $\mathrm{Y}$, finalmente, cerraremos con algunas reflexiones.

\section{La internalización de la educación superior}

Desde el ejemplo de Erasmo de Rotterdam que, debido a su extensa experiencia de movilidad académica ya en el siglo XV, inspira a los responsables europeos a fines de los ochenta del pasado siglo para bautizar con el nombre de Erasmus el principal programa de movilidad de estudiantes en Europa, muestra que la internacionalización ha existido desde hace larga data. Lo que se ha ido modificando es la intensidad del proceso, el volumen del intercambio de estudiantes, docentes e investigadores y, en los últimos años, sus objetivos. La educación superior tradicional, especialmente la universitaria, va a ser influenciada de diferentes maneras por estos distintos movimientos de apertura mundiales de intercambio de estudiantes e investigadores, por el movimiento de estudiantes y profesores, por las dinámicas migratorias, entre otros. La presencia de estudiantes, docentes o investigadores extranjeros en las distintas universidades del mundo ya representa parte de las situaciones cotidianas vividas en las distintas casas de estudios.

Desde el siglo XX y en apretada síntesis, es posible distinguir al menos tres momentos de la internacionalización. En primer lugar, durante la primera mitad del siglo XX la movilidad de profesores - especialmente de Europa a Estados Unidos- obedecía a razones extra-académicas, esto es, debido a los efectos de las dos guerras mundiales. En segundo lugar, a partir de la segunda mitad de ese siglo, la internacionalización comienza a expresarse vivamente en el contexto del paradigma del desarrollo, con una gran movilidad de estudiantes desde países "no avanzados" hacia los "avanzados" (dirección sur-norte). Dentro de este mismo contexto, surgen acuerdos de cooperación institucional en el sentido inverso (García Guadilla, 2005: 16). La movilidad era generada sobre todo por el deseo de los estudiantes y académicos de los "países pobres" de aprender, enseñar y llevar a cabo investigaciones en los "países ricos", para beneficiarse de la alta calidad de los sistemas de educación superior (SES) que ofrecían estos últimos, pero donde también los gobiernos intervenían fortificando o debilitando la movilidad y cooperación con países específicos, influenciando el proceso a través de distintos 
instrumentos: becas, premios, fondos para la investigación, visas, regulaciones, entre otros (Blumenthal et al., 1996: 2).

En tercer lugar, la última década del siglo XX se caracterizó, por un lado, por la afluencia de dinámicas integradoras de carácter regional amplio - como Europa y América Latina- y subregional - como el MERCOSUR - , además de los acuerdos regionales y los realizados entre instituciones. Por otro lado, paralelamente surge con bastante ímpetu una globalización del conocimiento que da un nuevo giro a la internacionalización, haciendo más evidente el aspecto lucrativo. Los cambios que marcan las profundas transformaciones a las que ha estado sometida la educación superior universitaria en el mundo entero, se inscriben en el auge del paradigma neoliberal, que implica nuevas pautas en la regulación de la educación superior cuyo telón de fondo ha sido la restructuración de la administración pública, que se produjo "con la adopción por parte de los gobiernos neoliberales del paradigma del 'nuevo gerenciamiento público' (new public management)" (Araujo, 2007: 70).

En los últimos años se aprecia una mayor relevancia de la internacionalización, debido a que ésta pasa a ser un elemento clave con el cual las instituciones académicas deben responder al impacto de la globalización (García Guadilla, 2005: 16-17). De todas formas, el flujo de intercambio de estudiantes e investigadores acentúa la tendencia: la movilidad internacional de estudiantes hacia los países de la Organización para la Cooperación y el Desarrollo Económicos (OCDE) se duplicó en los últimos 20 años. A mediados de los noventa, los países de la OCDE recibían cerca de $85 \%$ de estudiantes extranjeros del mundo entero. Europa constituye la principal región, pero Norteamérica es la más abierta a otras regiones tanto relativa como absolutamente, donde los asiáticos representan el $60 \%$ de los extranjeros (OCDE, 2004: 11). Entre 2000 y 2011, el efectivo mundial de estudiantes en formación en el extranjero hizo más que duplicarse con una tasa de crecimiento de más de 7\% anual promedio (OCDE, 2013: 313). En 2011, más de un estudiante extranjero sobre dos fue escolarizado en la enseñanza superior en Alemania, Australia, Canadá, Estados Unidos, Francia y Reino Unido. En valores absolutos, continúan siendo los Estados Unidos los que reciben la mayor cantidad de estudiantes extranjeros: $17 \%$ del efectivo mundial de estudiantes extranjeros - si bien en 2000 alcanzaban el 23\%-, seguidos del Reino Unido (13\%), Australia (6\%), Francia (6\%) y Canadá (5\%) (OCDE, 2013: 315).

Si bien la movilidad fisica es probablemente la imagen más fuerte y visible sobre la educación internacional, distintos factores han cambiado radicalmente la manera en la que la pensamos. Además de la movilidad individual, como señalan Skilbeck y Connell, necesitamos pensar en términos de movilidad de ideas, información, oportunidades, instituciones y programas para aprender (1996: 73). La movilidad de investigadores, docentes y estudiantes ha contribuido también con la circulación cognitiva y con la generación de diversos cambios institucionales en las diversas IES, ya sea en el desarrollo de la logística de recepción de esos académicos, o bien en la adaptación de esas instituciones a las dinámicas generadas por el flujo continuo de circulación de estos actores.

El fenómeno de globalización de la educación superior pareciera indicar que la tendencia se acentúa y diversifica. Por lo tanto, la internacionalización se desplaza de la periferia al centro de atención de las instituciones académicas y la movilidad académica se acentúa entre las redes definidas y reforzadas en términos de emulación de los modelos, no sólo atractivos cultural y académicamente, sino reconocidos como aquellos más compatibles con las demandas del mercado. A medida que gana importancia la internacionalización se vuelve más empresarial y entremezclada con procesos de comercialización, de competitividad, incluso para el caso de las universidades públicas. Como señala García Guadilla, "frente a esta tendencia de internacionalización 'lucrativa' es 
preciso favorecer opciones que apunten a una internacionalización cooperativa" (2005: 17).

Distintas instituciones compiten por la realización de acuerdos con universidades extranjeras que les permitan realizar intercambios con sus propios estudiantes. Como señalan algunos autores, "también existe un mercado de las posiciones académicas en el que las instituciones compiten por reclutar recursos humanos docentes. La competencia entre instituciones en estos mercados es una de las características salientes de la educación superior en el comienzo del nuevo milenio" (Landoni y Martínez, 2006: 3-4). Es decir, si en sus orígenes el aumento de la internacionalización se vinculaba a la idea de fomentar el intercambio académico-profesional y a la cooperación entre instituciones, en los últimos años está cada vez más dirigida a la competencia entre universidades, sectores de la educación superior de los diferentes países y regiones, razón por la cual algunos autores hablan de un cambio de paradigma de la internacionalización: de la cooperación a la competencia (De Wit, 2005: 222). En este sentido, en las últimas décadas se evidencia un marcado acercamiento entre los procesos de internacionalización y de mercantilización.

\section{La mercantilización y la competitividad en la educación superior}

El fenómeno de la internacionalización constituye una interconexión entre los sistemas públicos de enseñanza (Van der Wende, 2001), fuera de las relaciones comerciales donde la implementación no cuestiona la autoridad y los límites del Estado. Consecuentemente, el Estado juega un papel muy importante como mediador en las relaciones de cooperación entre les IES internacionales. En este sentido, las olas precedentes de internacionalización de las universidades se sitúan bajo la época colonial, cuando los imperios de España, Francia y Gran Bretaña introdujeron los primeros SES y sus instituciones en sus colonias (Charle y Verger, 2007; Verger, 2010: 61).
Como veremos a continuación, las diferencias del nuevo escenario internacional están dadas por tres fenómenos interrelacionados que han redundado en la profundización del proceso de mercantilización de los SES: en primer lugar, el aumento del intercambio económico que se traduce en nuevas normas internacionales que refuerzan los actores clave del comercio internacional, fenómeno que también se traducirá a nivel de los SES. En segundo lugar, la presencia de la lógica del mercado con sus demandas directas o indirectas hacia los SES, lo que se traduce en nuevos contenidos acordes con otras competencias y formaciones, que derivan en mayor competencia entre las IES, la proliferación de contenidos vinculados al mundo de los negocios, como la expansión de la oferta de masters of business administration (MBA), la delicada frontera entre la articulación universidad-empresa, la aparición de los rankings universitarios internacionales, etcétera. En tercer lugar, el aumento indiscriminado de las llamadas "universidades garaje" o "kiosco" (porque efectivamente pueden funcionar en un garaje, un viejo cine, un comercio, entre otros espacios físicos poco apropiados para la enseñanza y las actividades de investigación), de dudosa calidad académica, dedicadas excluyentemente a la "docencia" y orientadas a generar una alta rentabilidad en relación con la mínima inversión realizada.

En relación con el primer fenómeno, la aceleración de las dinámicas de transnacionalización que ha dado lugar a su traducción en acuerdos comerciales de libre comercio, donde los circuitos están diseñados para el consumo y la venta de "servicios" universitarios - a lo cual nos referiremos más puntualmente en la próxima sección-, independientemente de los límites del Estado o de otros factores condicionantes. La transnacionalización depende de la capacidad de las universidades para establecer sucursales en diferentes países sin restricciones, para expedir certificados reconocidos en otros lugares o contratar libremente a docentes e investigadores internacionales (Verger, 2010: 61). 
En relación con el segundo fenómeno, en un contexto cada vez más competitivo, alentado por la globalización económica y financiera, existen diversos incentivos, en particular por parte de las grandes empresas, a contratar y valorar positivamente a los profesionales capaces de adaptarse y, especialmente, comprender otros contextos. Así como también a aquellos predispuestos a trasladarse con el fin de trabajar en forma estable o transitoria en otros países, o bien a los que ya cuentan con una experiencia de vida en el extranjero, lo cual también constituye un incentivo adicional para la elección que realizan los estudiantes sobre sus posibles destinos. En este sentido, los intercambios universitarios funcionan como un incentivo para que los estudiantes consigan su primer empleo ya sea en su país de origen o en el de su estancia de formación. En general, al haber aprendido otro idioma, contar con una especialización o suplemento al diploma y al haber realizado una experiencia de vida en otro país, se encuentran en mejores condiciones para ser reconocidos por sus potenciales empleadores. En palabras de uno de nuestros entrevistados:

\begin{abstract}
El principal beneficio es que voy a saber hablar bien otros idiomas además del propio. Y el hecho de haber estudiado aquí me abre más las puertas para poder trabajar aquí. Si voy a una empresa y les digo he estudiado 6 meses en la Escuela de Comercio de Chambery, te dicen la Escuela de Chambery la conocemos, sabemos lo que es, sabemos cómo es este chico, pero si digo la Universidad de Zaragoza no quiere decir nada (Entrevista con un estudiante español de Erasmus en su estadía en Francia, 03-02-2010).
\end{abstract}

Desde los años noventa, una de las preocupaciones que afectan sobre todo a Europa es si la oferta educativa es demasiado académica para satisfacer las necesidades empresariales, y si se descuidan las competencias de gestión y control (Alpin y Shackleton, 1997), mientras que por su parte, el modelo estadounidense parecía haberse impuesto mejor y según los parámetros del mercado. La multiplicación de los MBA y su extensión incluso dentro de algunas de las universidades más prestigiosas de Europa, no vinculadas al mundo de los negocios, testimonia la necesidad de captar más graduados acompañados de financiamiento. Si en sus inicios la demanda se orientaba exclusivamente a los ejecutivos de importantes empresas y los altos costos de la formación eran absorbidos por los empleadores, actualmente este tipo de master se ha generalizado en América Latina como una moda, donde inclusive algunas universidades públicas lo contemplan entre su oferta de cursos de posgrado. Este ejemplo constituye una muestra evidente de cómo la cultura americana se generaliza en el ámbito de la educación superior al igual que en tantos otros. Como sostiene Edward Said, "muy pocas veces en el transcurso de la historia humana se ha producido una intervención tan masiva de la fuerza y las ideas de una cultura en otra como la que Norteamérica opera en la actualidad sobre el resto del mundo" (Said, 1993: 491).

$\mathrm{El}$ acercamiento que tiene lugar entre el mundo universitario y el de los negocios comenzó a reforzarse en EUA en los años ochenta, según Masseys-Bertonèche (2011), con la emergencia del tercer sector y la profesionalización de la filantropía. Allí los prestatarios con fines lucrativos y los centros de formación de las empresas son cada vez más activos y ganan terreno en las formaciones diplomadas, en principio pensadas sólo para la formación de sus propios empleados - como las universidades de McDonald y Motorola-, han multiplicado y diversificado su oferta de programas a fin de atraer una clientela mayor (Vincent-Lancrin, 2004: 26). Ello ha beneficiado en materia económica y de políticas a determinadas áreas en el seno de los SES en perjuicio de otras. Como sostiene Nussbaum en su crítica a un informe final sobre el futuro de la enseñanza superior en los Estados Unidos (Commission Appointed, 2006), demasiado concentrado sobre el beneficio económico: "Se interesa en los aparentes faltantes en ciencias, 
tecnología e ingeniería: no en las investigaciones científicas fundamentales, sólo en un aprendizaje extremamente técnico, capaz de engendrar rápidamente estrategias de búsqueda del beneficio. Las humanidades, las artes y el pensamiento crítico estaban ampliamente ausentes" (Nussbaum, 2011: 11).

Con la clasificación mundial de rendimiento de la investigación, la educación superior en sí ha entrado en una era de competencia global abierta entre las naciones y entre las IES individuales como actores globales en su propio derecho (Marginson y van der Wende, 2007: 307). De esta forma, la aparición de los distintos rankings universitarios, como el de Shanghai Jiao Tong, constituye otra de las caras de la competitividad interuniversitaria. Su instrumento de medición contribuye a reforzar esa carrera de la competitividad entre las IES e inspirar reformas de los SES tendientes a mejorar el posicionamiento de estas instituciones en el índice, a la vez que aquellas universidades mejor ubicadas constituyen la oferta más atractiva en las elecciones de destinos posibles que realizan los "estudiantes móviles".

La tendenciosa ponderación de la clasificación de Shanghai lleva a privilegiar ciertos indicadores que poco se vinculan con la calidad académico-científica de las universidades, mientras que se subvaloran otros esenciales para una completa evaluación de las ciencias humanas y sociales. Por poner dos ejemplos, la ponderación de un 20\% sobre los profesores galardonados con un premio nobel (en química, física, medicina, economía y matemáticas) ha incentivado la carrera entre algunas universidades para lograr cooptar estas preciadas figuras entre su staff. En relación con las ciencias sociales, se pondera con otro $20 \%$ solamente los artículos citados en revistas indexadas pero, por un lado, gran parte de ellas no figuran en sus listados y, por otro lado, por las características de las ciencias sociales, los libros y publicaciones colectivas donde se suelen volcar en mayor medida los resultados de proyectos individuales o colectivos de investigación no se tienen en cuenta. Como señala Linch, los principales beneficiarios del índice del Times y del de Shanghai es la industria de edición en lengua inglesa: clasificar las universidades proporciona informaciones que interesan y contribuye a aumentar considerablemente la venta de los diarios porque el lugar de la universidad en la clasificación depende fuertemente de las publicaciones en las revistas con referato, principalmente en inglés, que en general son controladas por multinacionales de la edición. Ello genera un círculo vicioso donde la clasificación aumenta la venta de publicaciones y de revistas a fondos privados (Linch, 2011), lo que hace que los investigadores busquen publicar en esos journals considerados los más prestigiosos y leídos, lo cual lleva, efectivamente, a consolidar el impacto de estos journals, que a su vez colaboran con el prestigio de su propia universidad y que, consecuentemente, aplaude el índice en cuestión (véase gráfica 1).

El movimiento de creación de rankings mundiales para las universidades simbólicamente constituye el indicador más poderoso que el mercado de valores ha incorporado en el sector universitario. Lo significativo es que el ranking es llevado a cabo por operaciones comerciales (en muchos casos periódicos) y universidades que tienen algún control sobre la operación (Linch, 2011: 28). En estos índices fuertemente cuestionados, las ciencias sociales "no rentables" prácticamente no son contabilizadas y en estructuras científico-tecnológicas nacionales, donde existen instituciones dedicadas exclusivamente a estas actividades por fuera de las universidades, toda esta actividad no se computa en favor de la actividad universitaria de estos países, como por ejemplo en los casos de Francia ${ }^{2}$ y Argentina.

\footnotetext{
${ }^{2}$ Sobre el índice de Shanghai resulta sumamente ilustrativo el documental "Universités, le grand soir" (Universidades, la gran noche), realizado por Thomas Lacoste y apoyado por los colectivos L'Autre campagne (La otra campaña) y Sauvons la recherche (Salvemos la investigación), disponible en: http://www.dailymotion.com/playlist/x7g6p_lautrecampagne_universites-le-grand-soir-lefilm/1\#video=x3fgh7.
} 


\section{Gráfica 1. El círculo vicioso del índice de Shanghái}

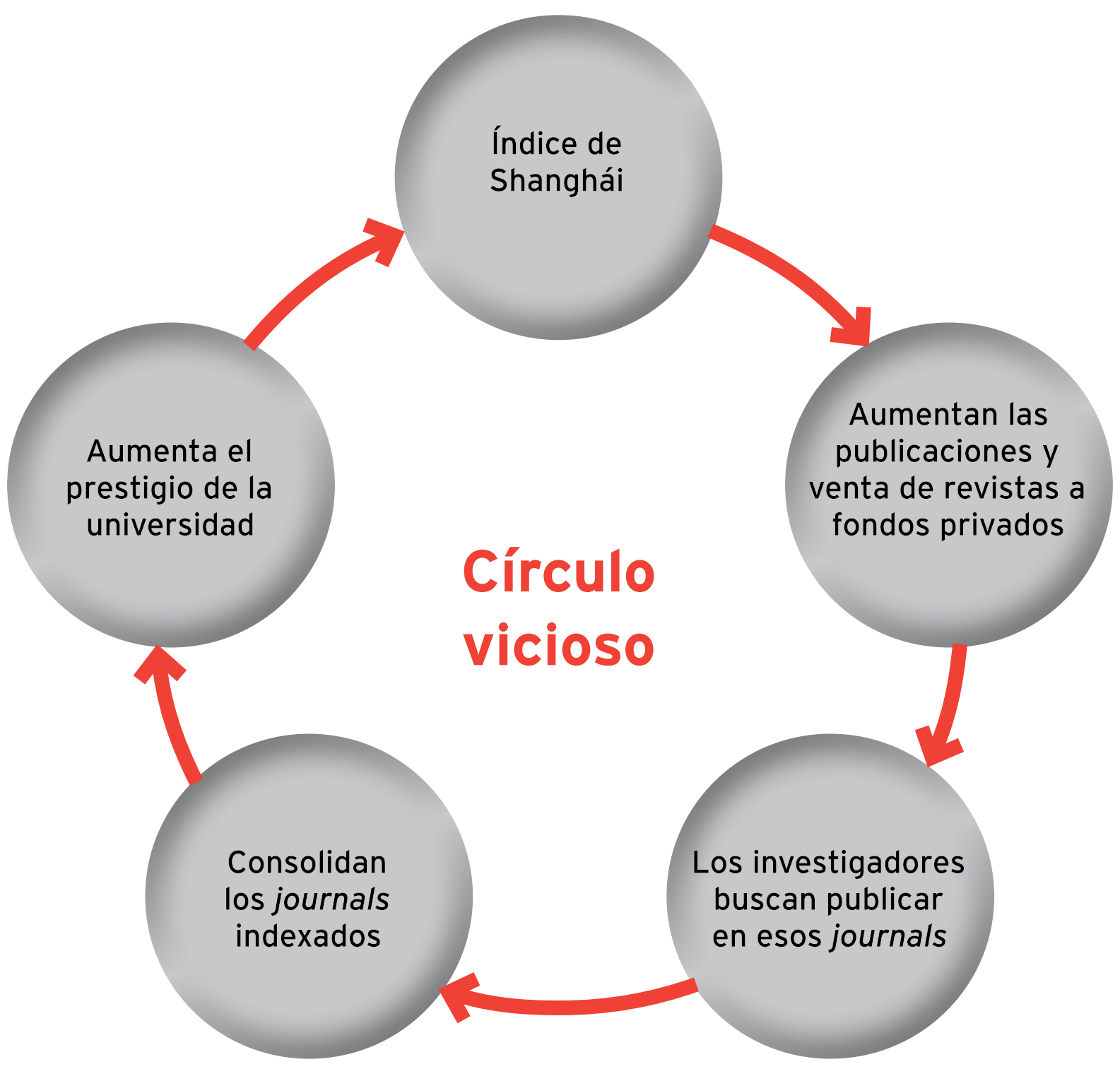

Con relación al tercer fenómeno aludido precedentemente, la multiplicación de la oferta universitaria y terciaria en América Latina ha obedecido en buena medida a la mercantilización de la educación superior, donde la oferta de IES apunta especialmente a carreras que requieren un bajo nivel de inversión para poder funcionar, lo cual constituye una diferencia importante con la UE. Los países del
MERCOSUR constituyen claros exponentes de esta tendencia, donde podemos observar (cuadros 1 y 2) cómo en menos de dos décadas se han multiplicado la cantidad de IES en estos cuatro países.

El caso de Brasil reviste además la particularidad de sus enormes dimensiones, donde los establecimientos aislados de enseñanza superior de carácter privado se han casi cuadruplicado en los últimos años (cuadro 3). 
Cuadro 1. Cantidad de universidades de los países del MERCosUR al momento de su fundación (1991)

\begin{tabular}{|l|c|c|}
\hline \multicolumn{1}{|c|}{ Países } & Públicas & Privadas \\
\hline Argentina & 35 & 35 \\
\hline Brasil & 55 & 40 \\
\hline Paraguay & 1 & 1 \\
\hline Uruguay & 1 & 1 \\
\hline
\end{tabular}

Fuente: ministerios de educación de los cuatro países.

Cuadro 2. Cantidad de universidades de los países del MERCOSUR en 2009

\begin{tabular}{|l|c|c|}
\hline \multicolumn{1}{|c|}{ Países } & Públicas & Privadas \\
\hline Argentina & 48 & 56 \\
\hline Brasil & 96 & 87 \\
\hline Paraguay & 7 & 38 \\
\hline Uruguay & 1 & 5 \\
\hline
\end{tabular}

Fuente: ministerios de educación de los cuatro países.

Cuadro 3. El caso de Brasil y los establecimientos aislados de enseñanza superior*

\begin{tabular}{|l|c|c|}
\hline \multicolumn{1}{|c|}{ Evolución / sector } & Público & Privado \\
\hline 1991 & 167 & 582 \\
\hline 2007 & 149 & 1829 \\
\hline
\end{tabular}

* Para el año 1991, hay que agregar 74 federaciones de facultades privadas y para 2007 la existencia de centros universitarios: 4 públicos y 116 privados.

Fuente: Instituto Nacional de Estudos e Pesquisas Educacionais/Brasil. 
El proceso de competencia entre IES tendrá importantes consecuencias en América Latina y en Argentina. Ante la proliferación de distintas IES privadas o "sucursales" de universidades internacionales que se ofrecen como "fábricas de títulos", en los años noventa empezó a discutirse el tema de los sistemas de control de la calidad y acreditación. La enorme expansión de instituciones de educación superior en América Latina y particularmente en Brasil, en estos últimos años, está interrelacionada con las olas generales de privatizaciones, mercantilización y la existencia de un casi-mercado educacional. Todo ello, junto con las políticas de flexibilización, constituye lo que se suele llamar "modernización", que no es otra cosa que la adaptación del sistema de educación superior al neoliberalismo económico y al neoconservadurismo político (Dias Sobrino, 2007: 314). No está demás señalar que en el marco de este proceso existen diversos claroscuros, extrañas formas de convivencia que atraviesan el eterno debate Estado versus mercado, así como distintas formas de procesamiento institucional de acuerdo con las diferentes tradiciones políticas y universitarias en el seno de los países de la región (Cf. Unzué y Emiliozzi, 2013).

Como consecuencia de este fenómeno de multiplicación de universidades y a fin de garantizar la calidad de las formaciones, en el caso del MERCOSUR se ha concentrado la atención sobre los mecanismos de acreditación universitaria, sobre los cuales se asientan los programas de movilidad académica regional.

\section{El papel de los organismos internacionales y la concepción de la educación como un servicio}

Las nuevas reglas internacionales promovidos por la OMC implican una redefinición de los territorios en relación con el suministro y consumo de los "servicios" de educación. El AGCS transforma la noción de servicios y el significado de lo que significa "servir" a la comunidad, un concepto de los servicios que se rigen por el mercado. En otras palabras, la educación está perdiendo su función social "especial" y se integra en la economía (Robertson et al., 2003: 10-11).

$\mathrm{El}$ primer documento que incorpora los "servicios educativos" dentro de las clasificaciones sectoriales de los servicios data de 1991 (OMC, 1991). Allí, los servicios de educación se clasifican en cinco categorías: servicios de educación primaria, de educación secundaria, de educación superior, de educación para adultos y una categoría residual que incluye otros servicios educativos. Los servicios de enseñanza superior objeto de este trabajo - incluyen los servicios de formación técnica y profesional postsecundaria, y otros servicios de enseñanza superior. La primera de estas clases se refiere a la enseñanza técnica y profesional, que no conduce a un título universitario y la segunda conduce a la obtención de un título universitario o diploma equivalente (OMC, 2010: 2). Al mismo tiempo, el documento final de la OMC, "los servicios de educación", hace hincapié en que "ciertos tipos de prestación más recientes provienen a menudo de dos modos de provisión o más y son difíciles de clasificar" (OMC, 2010: 10).

La fragmentación categórica, por sí misma, tiene importantes consecuencias prácticas. Como destaca Kesley, los compromisos asumidos en algún momento pueden tener consecuencias no deseadas cuando la naturaleza del servicio prestado o la identidad del distribuidor cambian. Estos compromisos confieren derechos a largo plazo a un contratista extranjero involucrado en uno de los aspectos socialmente desprendidos de un servicio. A pesar de que rara vez ocurre, en términos de protección de los derechos de estos proveedores, es extremadamente dificil reintegrar los servicios donde el enfoque desagregado falla, o si es conveniente adoptar un enfoque socialmente más centrado en los servicios básicos (Kesley, 2003: 275-276).

Resumiendo algunas de las consecuencias de la toma de compromisos en materia de "liberalización" de la educación superior en el seno del AGCS, éstos implicarían en principio que todos los beneficios o ventajas que puede percibir la educación superior de 
carácter privada al "comercializarse" y encontrarse en condiciones de "competencia" con otras IES, deben hacerse extensivos a todos los potenciales "proveedores" externos. Es decir, no es posible hacer una diferencia entre la enseñanza local y los "servicios" que pueden venir desde fuera y ofrecerse en el país, con lo cual si se subsidia al primero se debe subsidiar en iguales condiciones al segundo. Ahora bien, de aceptarse este tipo de compromisos, es difícil prever que en el futuro estos ámbitos de aplicación permanezcan reducidos a la esfera "privada" y no busquen amplificar sus "beneficios" como "prestadores" hasta alcanzar las mismas condiciones con que cuenta el sistema de enseñanza del ámbito público.

En los últimos años, como señala Zarur Miranda:

los diferentes "modos" de prestación del servicio educativo presentan dinámicas de acelerado crecimiento, especialmente el suministro transfronterizo, gracias a los desarrollos de la informática y las tecnologías de información y comunicaciones. Los hasta hace pocos años llamados "nuevos proveedores" hoy tienen más presencia y es cada vez más numerosa y variada la oferta y el tipo de cursos. Igualmente, la adquisición de instituciones ya establecidas en los países, ratifican el interés de los capitales por invertir en el "negocio de la educación superior", principalmente ante la expectativa de una creciente demanda en educación superior en la medida que se continúe dando mayor valor a la formación, por el papel que juega el conocimiento en los procesos productivos y en la competitividad, entre otros factores (2008: 184).

Bajo el neoliberalismo la educación es hoy un producto negociable. La presión de las nuevas ofertas en educación sobre los antiguos proveedores, seguros de su papel y de sus objetivos, los obligan cada vez más a utilizar las nuevas tecnologías para penetrar las fronteras, incrementalmente impulsadas por las actuales reglas mundiales del comercio hacia nuevos mercados. Los nuevos proveedores son empresas comerciales o semi-comerciales que producen identidades de marca que ellos utilizan globalmente. Son apoyados por los organismos de regulación internacional y por las economías fuertes (Lawn y Novoa, 2005: 42).

Si por un lado la concepción de servicios, acorde con la OMC, es avalada por los países desarrollados, América Latina ha rechazado de plano esta concepción. En la Conferencia Regional de Educación Superior de América Latina y el Caribe (CRES), celebrada en Cartagena del 4 al 6 de junio de 2008, se dejó asentada en su declaración final que la "Educación Superior es un bien público social, un derecho humano y universal y un deber del Estado". Asimismo, allí se advierte específicamente sobre los riesgos que entrañaría considerar a la educación como un servicio comercial, por lo cual se lo rechaza explícitamente (Solanas, 2009b), como se había venido reiterando en las distintas reuniones de Ministros de Educación del MERCOSUR a partir de 1999 (XVII ${ }^{a}$ RME ACTA № 2/99, punto 7), cuando el tema comenzó a instalarse en la agenda institucional de la región.

\section{La movilidad académica: la UE y el proceso de Bolonia, América Latina y el MERCOSUR}

A través de la localización de la internacionalización de la educación superior dentro de Europa, histórica y espacialmente se pueden observar cambios en su naturaleza, escala y alcance del proyecto geopolítico (Robertson, 2009: 78), aunque también existen importantes continuidades: la internacionalización ha continuado como actividad conducida por los Estados miembros. La discontinuidad con el pasado se concentra en la escala, el alcance y las consecuencias del proyecto de regionalización y globalización ideológica y política para la educación superior. Bajo el rubro de economía basada en el conocimiento, la educación superior es valorada por sus contribuciones económicas más que culturales, como un mercado lucrativo y como medios para generar nuevos valores a través de la innovación y las patentes (Robertson, 2009: 78). 
Es posible distinguir dos estrategias buscadas a lo largo del tiempo que usaban el discurso de internacionalización de la educación superior: la del regionalismo, construida en un conjunto coherente de estructuras que permiten un sistema europeo de educación superior y Europa viniendo a la existencia; la segunda más reciente, la de la globalización en educación superior, que es la fase actual. Ambas legitiman la Comisión Europea como actor en la etapa global y proveen un trampolín para el discurso y material europeo basado en la competencia con Estados Unidos por los corazones, mentes y bolsillos individuales en diferentes esferas del planeta (Robertson, 2009: 78).

El proceso intergubernamental de Bolonia iniciado en 1998 y cuyo objetivo principal era transparentar las formaciones europeas creando esquemas de educación superior legíblemente convergentes, se apoya técnicamente sobre uno de los instrumentos del Programa Erasmus: los ECTS. Sintetizando alguna de sus consecuencias, este sistema de créditos concebido a fines de traducir en unidades de contenidos transferibles los conocimientos adquiridos por los estudiantes en las universidades receptoras, servirían de base para proponer la transparencia y convergencia de las formaciones universitarias en el sistema L-M-D (por Licencia-Máster-Doctorado), donde las diferentes instancias abarcan 3-2-3 años respectivamente, correspondiendo a 60 créditos cada año de estudios. En palabras de uno de nuestros entrevistados, refiriéndose al caso francés:

los diplomas eran ilegibles. El diagnóstico de forma brusca era que solamente los franceses sabían que es un DEUG (diploma de 2 años de duración), mientras que en América todo el mundo sabe [lo que implica un diploma]. Había que encontrar una oferta legible, atractiva mundialmente (Entrevista con un ex funcionario del Ministerio de Educación de Francia, París, 02-07-2008).
Si previamente a Bolonia la movilidad académica tendía a privilegiar determinados destinos por sobre otros, con la puesta en marcha de este proceso se acentúa esta tendencia. Los países iniciadores del proceso aumentaron en mayor medida el ingreso de la movilidad entrante: Francia incrementó un $80 \%$, Alemania un $45 \%$, Gran Bretaña un $36 \%$ e Italia un $90 \%$ (OCDE, 2004).

Con el Proceso de Bolonia también aumenta el porcentaje de estudiantes provenientes de otros continentes: en los 45 países participantes del proceso, el $45 \%$ en promedio son estudiantes de otros continentes (OCDE, 2004). Aunque si bien actualmente Europa representa el primer destino para los estudiantes que siguen una formación de nivel terciario en un país en el que no residen - recibe el $48 \%$ de los estudiantes en movilidad internacional-, no representa la región más dinámica: desde 2000, el número de estudiantes en movilidad internacional se triplicó en Oceanía, pese a que la región recibe menos del $10 \%$ de todos los estudiantes extranjeros (OCDE, 2013: 313). La dirección de la movilidad es más probable que sea hacia los países más ricos, aquellos con las universidades con mejores rankings, y a los países occidentales en general; por lo tanto, desde una perspectiva nacional, este movimiento profundiza la repartición entre ganadores y perdedores en términos de los mejores talentos disponibles y captados por determinados países por sobre otros. En un informe, el Banco Mundial argumenta con razón - sin ninguna referencia al Proceso de Bolonia-, que la movilidad internacional de recursos humanos calificados continuará presentando "riesgos a largo plazo para inversiones en educación terciaria en muchas naciones" (World Bank, 2002: 19).

Como señala Kwiek, el tema de la movilidad intra-europea no es controvertido en los países más ricos, dado que el nivel de la enseñanza superior es similar y la movilidad entrante y saliente entre ellos es relativamente equilibrada en comparación con los nuevos países miembros de la UE. En el caso 
de los SES "menos avanzados" y los países "más pobres" de la región, el aumento de la movilidad de estudiantes puede convertirse en una vía de escape fácil que conduce a la fuga de cerebros permanente, tema sobre el cual existe una vasta literatura, en especial en América Latina (Cf. Pellegrino, 2000; Albornoz y otros, 2002; García de Fanelli, 2008; Didou Aupetit y Gerard, 2009; Luchilo, 2011). Esto no es una cuestión teórica: desde hace unos años la UE está muy preocupada por los jóvenes investigadores y estudiantes de doctorado que salen de Estados Unidos y que en su mayoría no volverá a Europa (Kwiek, 2004: 770; OCDE, 2002; Comisión Europea, 2003). De hecho, la comisaria europea responsable de educación y cultura, Viviane Reding, presentó las bases para hacer de la UE una prominente figura en el mercado mundial de la educación, argumentando que los gobiernos nacionales solos no pueden reunir los cambios de la globalización, las nuevas tecnologías y el simple mercado. Esto incluía un nuevo rol para el sector privado. Como señala Robertson, desde 2003 una serie de iniciativas de programas se han puesto en marcha, caracterizados por un fuerte énfasis en la competitividad global y el desarrollo de un mercado europeo de educación superior. Las iniciativas incluían el reclutamiento de los talentos de todo el mundo (Erasmus Mundus), la promoción de la idea de Bolonia global a través de Tuning y el proyecto Asia-Link, y la creación del mercado y la industria europeos de la educación superior (Robertson, 2009: 75). Erasmus Mundus constituye un intento de reclutar las mentes más brillantes alrededor del mundo, se puso en marcha junto con las exhortaciones a la movilización del capital intelectual de Europa. La esperanza es que los mejores talentos del extranjero puedan ser retenidos en la UE, y que el gasto europeo en maestrías, en investigación o programas de formación, van a generar una mayor actividad de investigación en toda la región (Robertson, 2009: 76).

Distintos cambios generacionales evidencian una modificación incremental buscando compatibilizar estos programas, que junto con el proceso de Bolonia se han globalizado, con la estrategia del Consejo Europeo de Lisboa de 2000, en busca de una Europa más competitiva. Esta estrategia marcó un punto de inflexión en la historia de la cooperación en educación a nivel europeo (Pépin, 2011: 25), buscando modernizar los sistemas educativos para convertirlos en una referencia de calidad mundial y a la UE en la economía del conocimiento más competitiva y dinámica del mundo para 2010. En ese año y en una sintonía similar se lanza "Europa 2020: una estrategia para un crecimiento inteligente, sostenible e integrador", como continuación de la estrategia de Lisboa, aunque dando cuenta de un contexto más complejo y buscando subsanar los supuestos déficits de implementación de la primera.

Por otra parte, se han escuchado distintas voces críticas en relación con algunas de las políticas de cooperación de la UE hacia América Latina. Entre ellas, en relación con el programa Tuning, Aboites señala que se trata de un proyecto trasplantado: la primera implicación del modelo Tuning es que se trata de un modelo desarrollado en Europa, pero que ya se impulsa sin mayores cambios en América Latina. En el 2001 este modelo universitario se consolidó en Europa y de inmediato, en el 2002, un pequeño grupo de representantes de universidades europeas y latinoamericanas del Espacio Común de Enseñanza Superior de la UE, América Latina y el Caribe (UEALC) aprueba adoptar la propuesta europea e impulsar su aprobación ante los órganos europeos bajo el nombre de Proyecto Tuning-América Latina (2010: 32). Efectivamente, para participar de este programa las universidades de América Latina incorporan el sistema ECTS europeo, para poder "validar" las "competencias" adquiridas en la universidad receptora y viceversa. Por lo tanto, el incentivo a la movilidad interregional estimula el proceso de transferencia del instrumento clave del programa Erasmus, con la consecuente "creditización a la europea" de los contenidos aprendidos. 
Aunque la movilidad no se limita sólo a los países desarrollados, existen distintas experiencias impulsadas por los países latinoamericanos, entre las que podemos mencionar las primeras iniciativas del Programa de Intercambio y Movilidad Académica (PIMA) de la Organización de Estados Iberoamericanos para la Educación, la Ciencia y la Cultura (OEI) hacia fines de los noventa, los programas Escala Estudiantil de la Asociación de Universidades Grupo Montevideo (AUGM), en cooperación con la OEI y, en los últimos años, cabe destacar el Programa de Movilidad Académica Regional para los cursos acreditados por el mecanismo de acreditación de carreras de grado en el MERCOSUR (MARCA), que fomenta la movilidad de los estudiantes de las carreras acreditadas a través del sistema ARCUSUR. En agosto de 2006 se lanzaba la primera experiencia piloto que involucraba a 17 de las 18 carreras de agronomía ya acreditadas, con un total de 85 estudiantes en movilidad (cinco por cada carrera).

A pesar de que los impactos del MARCA en términos de movilidad académica resultan muy acotados como para generar una masa crítica de estudiantes en movilidad en el MERCOSUR, tanto la cantidad de estudiantes, carreras, universidades y países participantes no ha dejado de crecer desde su creación (véase cuadro 4). Actualmente participan en este programa 20 universidades nacionales de Argentina, 15 de Bolivia, 25 de Brasil, 4 de Chile, 1 de Paraguay, 2 de Uruguay y 3 de Venezuela, de acuerdo con la convocatoria de 2013. La movilidad de estudiantes concierne a las carreras de agronomía, arquitectura, ingeniería (agronómica, civil, de materiales, electricista, electromecánica, electrónica, industrial, mecánica y química), medicina, veterinaria, enfermería y odontología. ${ }^{3}$

\section{Cuadro 4. Programa MARCA: movilidad académica regional para carreras acreditadas por el mecanismo de acreditación del MERCoSUR, convocatoria 2013}

\begin{tabular}{|l|c|c|}
\hline \multicolumn{1}{|c|}{ Países / estudiantes } & Enviados & Recibidos \\
\hline Argentina & 160 & 69 \\
\hline Bolivia & 100 & 147 \\
\hline Brasil & 115 & 7 \\
\hline Chile & 8 & 0 \\
\hline Paraguay* & 0 & 15 \\
\hline Uruguay & 16 & 7 \\
\hline Venezuela & 13 & $\mathbf{4 1 2}$ \\
\hline Total & $\mathbf{4 1 2}$ & 75 \\
\hline
\end{tabular}

* Paraguay no ha participado de esta última convocatoria, dada su temporaria exclusión política del bloque, aunque lo ha hecho en las convocatorias anteriores.

Fuente: Sector Educativo del MERCOSUR.

\footnotetext{
${ }^{3}$ Para mayor información sobre el MARCA, véase: http://programamarca.siu.edu.ar/index.php
} 
Al haber sido concebido como un proyecto articulado con las acciones de acreditación que viene implementando el MERCOSUR, el MARCA constituye una estratégica vinculada con el modelo regionalista, precedentemente evocado, más que una estrategia convergente con el modelo de la globalización de los SES. En este sentido, y a pesar de que tanto en el MERCOSUR como en América Latina se ha rechazado la concepción de servicios de la OMC, la región no es ajena al proceso de mercantilización de la educación superior, una de cuyas facetas, como veíamos previamente, ha sido su traducción en el aumento indiscriminado de la cantidad de IES. Ahora bien, iniciativas como el MARCA, diseñadas y generadas desde la región sin reeditar modelos preestablecidos, constituyen formas interesantes de construir los procesos de movilidad académica en el MERCOSUR, escapando de la lógica mercantil predominante a nivel global.

A diferencia del Tuning para América Latina, por un lado, el MARCA se basa en el mecanismo de acreditación regional diseñado por el MERCOSUR y, en consecuencia, la movilidad estudiantil se produce entre las carreras universitarias ya acreditadas, excluyendo de esta forma a las "universidades garages". Y, por otro lado y por ende, no se sustenta sobre el sistema de créditos europeo sino que se basa en un esquema propio.

De esta manera, podemos apreciar que, a diferencia de la UE, donde la redefinición de programas de movilidad, como el Erasmus Mundus, apuntan a captar recursos humanos de otras partes del mundo con un claro objetivo comercialista, predominando el paradigma de la competitividad, en América Latina y el MERCOSUR los programas que han ido surgiendo en los últimos años responden a los clásicos esquemas de cooperación internacional e intercambio universitario, donde los objetivos de índole económica constituirían una posible consecuencia indirecta de estas acciones y no el fin orientador de las mismas.

\section{Reflexiones finales}

La movilidad académica y los sistemas de educación superior (SES) no son ajenos al contexto donde se insertan. La exacerbación de ciertas tendencias comercialistas en torno a ellos es consecuencia directa del paradigma neoliberal predominante durante los últimos años, y de las transformaciones y efectos directos e indirectos que ha provocado. Si el principal objetivo de la movilidad consistía en el enriquecimiento del estudiante, docente o investigador a partir del intercambio científico y cultural en el extranjero, a partir del auge del neoliberalismo la movilidad le proporciona al estudiante las condiciones requeridas por el mercado para encontrar más fácilmente empleo, luego de su experiencia en el extranjero, probablemente en una empresa multinacional o en el país de la universidad receptora, mientras que por su parte los centros de investigación y universidades suelen ofrecer ventajosas condiciones de trabajo y de vida a los docentes e investigadores que reclutan temporalmente, con lo cual muchos terminan quedándose en el país receptor acentuando la tendencia a cooptar universitarios ya formados en los países de origen.

Los flujos de movilidad constatan que América Latina, pero sobre todo Asia (OCDE, 2013), exporta recursos humanos formados que, como parte de la estrategia europea, estarían remplazando su propia fuga de "materia gris" hacia Estados Unidos. En este sentido, tanto el programa Tuning como el Erasmus Mundus impulsan la creditización de los SES en América Latina, en sintonía con las necesidades del viejo continente. Los riesgos de este modelo de movilidad se vinculan con la expansión del paradigma de la competitividad y la mercantilización en materia de SES, que es acompañado por el proceso de Bolonia, el cual se inserta y converge con la estrategia europea de Lisboa y Europa 2020. La expansión de estos efectos a otras regiones lleva a potenciar el eje sobre los aspectos más comercialistas de la educación con graves consecuencias para su desarrollo. Como señala Aboites: 
la mercantilización de la educación priva al país de la existencia de grandes espacios culturales y de discusión sobre el futuro del país. Ésta será en todo caso cada vez más una discusión entre una reducida élite, porque aun aquellos pocos que podrán ingresar a la educación superior la verán transformada en un centro de capacitación superior para el trabajo, enajenada respecto de las necesidades de pertenencia, es decir, de cultura y de participación, que es la que finalmente da el sustento y la trabazón más importantes a un país (2004: 6).

\section{Referencias}

Aboites, Hugo (2010), "La educación superior latinoamericana y el proceso de Bolonia: de la comercialización a la adopción del Proyecto Tuning de competencias", en Educación Superior y Sociedad, núm. 1, pp. 25-44.

Aboites, Hugo (2004), "Derecho a la educación o mercancía: la experiencia de diez años de libre comercio en la educación mexicana”, en Memoria. Revista Mensual de Política y Cultura, núm. 187, septiembre.

Albornoz, Mario, Lucas Luchilo, Gustavo Arber, Rodolfo Barrere y Julio Raffo (2002), "El talento que se pierde. Aproximación al estudio de la emigración de profesionales, investigadores y tecnólogos argentinos", documento de trabajo, Centro Redes, <http://www. centroredes.org.ar $>$ [consulta: agosto de 2013].

Alpin, Carmen y J. R. Shackleton (1997), “Tendencias en el mercado de trabajo y necesidades de información: sus efectos sobre las políticas de personal”, en Formación Profesional, núm. 12, CEDEFOP, septiembre-diciembre/ III, pp. 7-14.

Araujo, Sonia Marcela (2007), "Evaluación institucional y cambio universitario. Un dificil proceso de reconstrucción", en Pedro Krotsch, Antonio Camou y Marcelo Prati
Para terminar, otras experiencias de movilidad académica, como el mencionado programa MARCA, constituyen esfuerzos de integrar la región a partir de esquemas propios, aunque para expandir sus impactos y generar una masa crítica regional, sería preciso aumentar la escala de intercambios realizados en América Latina y el MERCOSUR.

(coords.), Evaluando la evaluación: políticas universitarias, instituciones y actores en Argentina y América Latina, Buenos Aires, Prometeo Libros, pp. 69-94.

Bhandari, R. y Peggy Blumenthal (eds.) (2011), International students and global mobility in higher education, Nueva York, Palgrave Macmillan.

Blumenthal, Peggy, Crauford Goodwin, Alan Smith y Ulrich Teichler (eds.) (1996), Academic mobility in a changing world. Regional and global trends, London and Bristol, Pennsylvania, Jessica Kingsley Publishers.

Charle, Christophe y Jacques Verger (2007), Histoire des universités, París, PUF, 1994, 2ème ed.

Chiroleu, Adriana (2000), "La educación superior en los países del MERCOSUR. El reconocimiento de las singularidades como punto de partida de la integración regional", en Revista de la Educación Superior, vol. XXIX, núm. 115, ANUIES.

Commission Appointed by Secretary of Education Margaret Spellings (2006), "A test of leadership charting the future of U. S. higher education", disponible en: <http://www.ed.gov/about/bdscomm/list/hiedfuture/ index.html> [consulta: febrero de 2012].

Dias Sobrinho, José (2007), "Evaluación de la educación 
superior en Brasil: la cuestión de la calidad", en Pedro Krotsch, Antonio Camou y Marcelo Prati (coords.), Evaluando la evaluación: políticas universitarias, instituciones y actores en Argentina y América Latina, Buenos Aires, Prometeo Libros, pp. 311-329.

Didou Aupetit, Sylvie y Etienne Gerad (eds.) (2009), Fuga de cerebros, movilidad académica y redes científicas. Perspectivas latinoamericanas, México, IESALC/CINVESTAV/IRD.

Didou Aupetit, Sylvie (2007), "La internacionalización de la educación superior en América Latina: oportunidades y desafíos", Secretaría de Asuntos Académicos, Universidad Nacional de Córdoba, Conferencia.

Didou Aupetit, Sylvie (2006), "Internacionalización de la educación superior y provisión transnacional de servicios educativos en América Latina: del voluntarismo a las elecciones estratégicas", Seminario Internacional IESALC-UNESCO/Conferencia de Rectores, Panamá, 16-17 marzo.

De Wit, Hans (2005), "América Latina y Europa ante el fenómeno de la internacionalización", en José Ginés Mora y Norberto Fernández Lamarra (coords.), Educación superior, convergencia entre América Latina y Europa [Procesos de evaluación y acreditación de la calidad], Caseros, Universidad Nacional de Tres de Febrero.

ESU (2012), Bologna With Student Eyes 2012, Brussels, en: <http://www.esu-online.org/news/article/6068/ Bologna-With-Student-Eyes-2012/> [consulta: septiembre de 2013].

European Commission (2003), Third European Report on Science \& Technology Indicators. Towards a Knowledge-Based Economy, Luxemburgo, Office for Official Publications. García de Fanelli, Ana María (2008), "Políticas públicas frente a la 'fuga de cerebros': reflexiones a partir del caso argentino", en Revista de la Educación Superior, vol. XXXVII (4), núm. 148, octubre-diciembre, ANUIES, pp. $111-121$.

García Guadilla, Carmen (2005), “Complejidades de la globalización e internacionalización de la educación superior. Interrogantes para América Latina", en Cuadernos del CENDES, año 22, núm. 58, tercera época enero-abril, pp. 1-22.
Haug, Guy(2010), "Lainternacionalización delaeducación superior: más allá de la movilidad europea", en $L a$ Cuestión Universitaria, núm. 6, pp. 20-29.

Hermo, Javier y Cecilia Pittelli (2008), "Globalización e internacionalización de la educación superior. Apuntes para el estudio de la situación en Argentina y el MERCOSUR", en Revista Española de Educación Comparada, núm. 14, pp. 243-268.

Kelsey, Jane (2003), "Legal fetishism and the contradictions of the GATS", en Globalisation, Societies and Education, vol. 1, núm. 3, noviembre, pp. 267-280.

Krotsch, Pedro, Antonio Camou y Marcelo Prati (coords.), Evaluando la evaluación: políticas universitarias, instituciones y actores en Argentina y América Latina, Buenos Aires, Prometeo Libros, pp. 69-94.

Kwiek, Marek(2004), “The emergent european educational policies under scrutiny: the Bologna Process from a central european perspective", en European Educational Research fournal, vol. 3, núm. 4.

Landoni Couture, Pablo y Enrique Martínez Larrechea (2006), "La institucionalidad de la acreditación: ¿hacia dónde vamos? La autonomía universitaria y el rol del Ministerio de Educación”, Montevideo, Instituto Internacional para la Educación Superior en América Latina y El Caribe (IESALC/UNESCO), Consejo de Rectores de Universidades de Uruguay, 5 y 6 de abril.

Lawn, Martin y António Novoa (coords.) (2005), L'Europe réinventée. Regards critiques sur l'espace européen de l'éducation, París, L'Harmattan.

Leher, Roberto (2009), "Estrategias de mercantilización de la educación y tiempos desiguales de los tratados de libre comercio: el caso de Brasil", en Pablo Gentili, Gaudêncio Frigotto, Roberto Leher y Florencia Stubrin (comps.), Políticas de privatización, espacio público y educación en América Latina, Rosario, CLACSO/Homosapiens Ediciones.

Linch, Kathleen (2011), "International league tables and rankings in higher education: an appraisal", en Imelda Elliott, Michael Murphy, Alain Payeur y Raymond Duval (dir.), Mutations de l'enseignement supérieur et internationalisation. Change in higher education and globalisation, 
Bruselas, De Boeck, pp. 19-44.

Luchilo, Lucas (2011), Más allá de la fuga de cerebros. Movilidad, migración y diásporas de argentinos calificados, Buenos Aires, EUDEBA-REDES.

Marginson, Simon y Marijk van der Wende (2007), "To rank or to be ranked: the impact of global rankings in higher education", en Journal of Studies in International Education, vol. 11, núm. 3-4, otoño-invierno, pp. 306-329.

Marquina, Mónica (2006), "La evaluación por pares en el escenario actual de aseguramiento de la calidad de la educación superior", Buenos Aires, CONEAU/ Ministerio de Educación, Ciencia y Tecnología.

Masseys-Bertoneche, Carole(2011), "Lacommercialisation des universités américaines: exemplarité pour l'Europe?", en Imelda Elliott, Michael Murphy, Alain Payeur y Raymond Duval (dir.), Mutations de l'enseignement supérieur et internationalisation. Change in higher education and globalisation, Bruselas, De Boeck, pp. 129-144.

Meabe, Joaquín E. y Eduardo R. Saguier (2011), La mano invisible del mercado en la educación y la ciencia. Historia crítica de una tragedia cívico-cultural argentina, Buenos Aires, Corrientes, <http://www.ses.unam.mx/documentos/ LaManoInvisibleDelMercado.pdf $>$ [consulta: julio de 2012].

MERCOSUR/RME (1999), XVII Reunión de Ministros de Educación de los Países Signatarios del Tratado del Mercado Común del Sur, ACTA 2/99, Montevideo, 26 de noviembre.

Musselin, Christine (2008), "Vers un marché international de l'enseignement supérieur ?", en Critique internationale, núm. 39, pp. 13-24.

Nussbaum, Martha (2011), Les émotions démocratiques: comment former le citoyen du XXIe siècle? [traduit de l'anglais (Etats-Unis) par Solange Chavel], París, Climats, impr. OCDE (2013), Regards sur l'éducation 2013: Les indicateurs de l'OCDE, Ediciones OCDE, <http://www.keepeek.com/ Digital-Asset-Management/oecd/education/regards-surl-education-2013/indicateur-c4-qui-etudie-a-l-etrangeret-ou_eag-2013-23-fr> [consulta: julio de 2013].

OCDE (2004), Enseignement supérieur: internationalisation et commerce, París, OCDE.
OCDE (2002), OECD Science, Technology and Industry Outlook, París, OCDE.

OMC (1991), "Classification sectorielle des services. Note du secrétariat", Restricted, MTN.GNS/W/120, Distribution spéciale, 10 juillet.

OMC, Conseil du commerce des services (2010), "Services d'éducation", Note d'information du Secrétariat, Restricted, $\mathrm{S} / \mathrm{C} / \mathrm{W} / 313$, ler avril.

Pellegrino, Adela (2000), "Éxodo, movilidad y circulación: nuevas modalidades de la migración calificada", <http://socinfo.eclac.org/publicaciones/xml/2/8852/ lcg2124P_4.pdf> [consulta: enero de 2013].

Pépin, Luce (2011), "Education in the Lisbon strategy: assessment and prospects", en European fournal of Education, vol. 46, núm. 1, pp. 25-35.

Plotkin, Mariano Ben (2006), La privatización de la educación superior y las ciencias sociales en Argentina. Un estudio de las carreras de Psicología y Economía, Buenos Aires, CLACSO.

Rama, Claudio (2012), "El negocio universitario for-profit América Latina", en Revista de la Educación Superior, vol. XLI, núm. 4, octubre-diciembre, pp. 59-95.

Robertson, Susan (2009), "Europe, competitiveness and higher education: an evolving project", en Roger Dale y Susan Robertson (eds.), Globalisation and europeanisation in education, Oxford, Symposium Books, pp. 65-83.

Robertson, Susan (2003), "WTO/GATS and the global education services industry", en Globalisation, Societies and Education, Vol. 1, núm. 3, pp. 259-266.

Robertson, Susan L. y Dale Roger (2003), "Changing geographies of power in education: the politics of rescaling and its contradictions", published by the Centre for Globalisation, Education and Societies, University of Bristol, Bristol BS8 1JA, UK, <http:// www.bris.ac.uk/education/people/academicStaff/edslr/ publications/03slr/> [consulta: septiembre de 2011].

Saforcada, Fernanda (2009), "Alambrando el bien común: conocimiento, educación y derechos sociales en los procesos de privatización y mercantilización de las últimas décadas", en Pablo Gentili, Gaudêncio Frigotto, Roberto Leher y Florencia Stubrin (comps.), Políticas de privatización, espacio público y educación en América 
Latina, Rosario, CLACSO/Homosapiens Ediciones.

Said, Edward W. (1993), Cultura e imperialismo, Barcelona, Anagrama.

Skilbeck, Malcolm y Helen Connell (1996), "International education from the perspective of emergent world regionalism", en Blumenthal et al., Op. Cit., pp. 66-102.

Solanas, Facundo (2012), "El Estado acreditador: del caso argentino al MERCOSUR", en Denise Leite, Maria Elly Herz Genro, Facundo Solanas, Vivian Fiori y Raúl Alberto Álvarez Ortega, Políticas de evaluación universitaria en América Latina: perspectivas criticas, Buenos Aires, CLACSO/IIGG, pp. 99-138.

Solanas, Facundo (2009a), "La Ley de Educación Superior en Argentina: un análisis en términos de referenciales de la acción pública", en Revista de la Educación Superior, vol. XXXVIII (1), núm. 149, enero-marzo, ANUIES, pp. 155-170.

Solanas, Facundo (2009b), "El impacto del MERCOSUR en la educación superior: un análisis desde la 'Mercosurización' de las políticas públicas”, en Archivos Analíticos de Políticas Educativas, vol. 17, núm. 20, octubre, $<$ http://epaa.asu.edu/epaa/> [consulta: septiembre de 2009].
Unzué, Martín y Sergio Emiliozzi (comp.) (2013), Universidad y politicas públicas ¿En busca del tiempo perdido? Argentina y Brasil en perspectiva comparada, Buenos Aires, Imago Mundi.

Verger,Antoni(2010),WTO/GATSand theglobalpolitics of higher education, Nueva York, Routledge, 248 páginas.

Vincent-Lancrin, Stéphan (2004), "L'enseignement transnational: une vue d'ensemble", en OCDE, Enseignement supérieur : internationalisation et commerce, París, OCDE, pp. 1944.

Wende van der, Marijk C. (2001), "The international dimension in national higher education policies: what has changed in Europe over the last five years?", en European Fournal of Education, vol. 36, núm. 4, 2001, 431-441.

World Bank (2002) Constructing knowledge societies: new challenges for tertiary education, Washington, DC, World Bank.

Zarur Miranda, Xiomara (2008), "Integración regional e internacionalización de la educación superior en América Latina y el Caribe”, en Lucia Gazzola y Axel Didriksson (eds.), Tendencias de la educación superior en América Latina y el Caribe, Caracas, IESALC-UNESCO.

\section{Cómo citar este artículo:}

Solanas, Facundo (2014), "Intercambio cooperativo versus mercantilización competitiva: las políticas de movilidad académica en el MERCOSUR y la Unión Europea", en Revista Iberoamericana de Educación Superior (RIES), México, UNAM-IISUE/Universia, vol. V, núm. 12, pp. 3-22, http://ries.universia.net/index.php/ries/article/ view/440 [consulta: fecha de última consulta]. 